\title{
Health concerns associated with travelling with inflammatory bowel disease (IBD): a questionnaire survey
}

\author{
Authors: Varun Philip, ${ }^{A}$ Anet Soubieres ${ }^{B}$ and Andrew Poullis ${ }^{C}$
}

When travelling, patients with inflammatory bowel disease (IBD) have a higher risk of morbidity. We identified barriers to travel, specific health concerns and several areas for service improvement among patients. In total, 136 patients were given a 32-question service improvement questionnaire. Of these, $89 \%$ travelled abroad, $30 \%$ reported that IBD limited travel and $40 \%$ said it affected choice of destination. Fourty-seven percent of patients travelled abroad without health insurance and $7 \%$ were refused. Seventy-eight percent wanted pre-travel advice from doctors in the future. Popular service improvement options included providing written prescriptions (91\%) and management plans (75\%). Sixty-three percent of patients were unaware of needing to avoid live vaccines while on immunosuppressants. Ninety-two percent were unaware that high altitudes could precipitate flares; $27 \%$ travelled abroad to high-altitude destinations, of which $46 \%$ subsequently had flare-ups. Existing IBD travel services remain unknown such as the 'Can't wait' card (72\%) and 'IBD passport' (96\%). Service improvements in the IBD clinic need to be implemented to facilitate safer travel overseas.

KEYWORDS: Inflammatory bowel disease, travelling, health concerns, service improvement, immunosuppression

\section{Introduction}

Patients with inflammatory bowel disease (IBD) who travel abroad are at higher risk of travel-related morbidity for a range of reasons. ${ }^{1}$ Firstly, travelling abroad in itself may trigger an exacerbation of IBD otherwise known as a 'flare-up'. A multitude of factors such as mode of travel, climate/altitude of destination, diet and varying sanitation standards could proffer explanations as to why flareups occur. Secondly, IBD patients travelling abroad have a higher predisposition to acquiring bowel infections ${ }^{2}$ which are significantly more likely while receiving immunosuppressant medication.

It is important that patients on immunosuppressants are informed by their physician to avoid any 'live' vaccines, such as the

Authors: Afoundation doctor, St George's University of London, London, UK; ' ' gastroenterology research registrar, St George's University of London, London, UK; ' Consultant gastroenterologist, St George's University of London, London, UK yellow fever vaccination. An alarmingly high number of patients are uninformed and undergo inappropriate vaccination. Live vaccines need to be avoided in patients on immunosuppressants and non-live vaccines may induce a reduced immune response and have reduced efficacy. ${ }^{3}$

Travelers with IBD often have to pay excessive premiums to secure health insurance when travelling abroad. Patients are sometimes even refused health insurance altogether ${ }^{4}$ and forced to travel uninsured which puts them at risk of being unable to access healthcare. This project aims to investigate all the factors detailed above and to ascertain the extent at which they affect patients in the general population. In response to patients' problems we sought to identify areas of service improvement which alleviate concerns and facilitate easy travel in the future.

\section{Methods}

As part of an outpatient service improvement project a 32-question paper-based questionnaire was designed and distributed in three weekly clinics at St George's Hospital, London. The three clinics used were an IBD specialist doctor clinic, IBD specialist nurse clinic and an IBD infusion clinic where patients receive anti-tumour necrosis factor (TNF) treatment. Patients of all ages were selected with the only qualifying criteria being a current diagnosis of IBD. Convenience sampling was used to recruit participants. Sampling questions asked for details of the patient's gender, age, type of disease and the date of diagnosis. Although travel abroad was preferable, patients were not excluded from the study if they had never travelled abroad.

The purpose of the study was to explore patient attitudes towards travelling and to identify areas of unmet need in the IBD service. The survey also investigated whether or not a diagnosis of IBD affects travel plans and is a hindrance to patients, as the literature suggests. The choice of answers available for most questions consisted of 'yes', 'no' and 'non-applicable' to ensure a high yield of quantitative data. Questions which addressed areas of service improvement included multiple options with the instruction to 'Tick all that apply'. Ethics committee approval was not required according to the UK National Research Ethics Service. Informed consent was obtained.

\section{Results}

Results were obtained from 136 patients aged 18-81 years old. The mean age in the sample was 40 years. The gender distribution was approximately equal with 70 males and 66 females being 


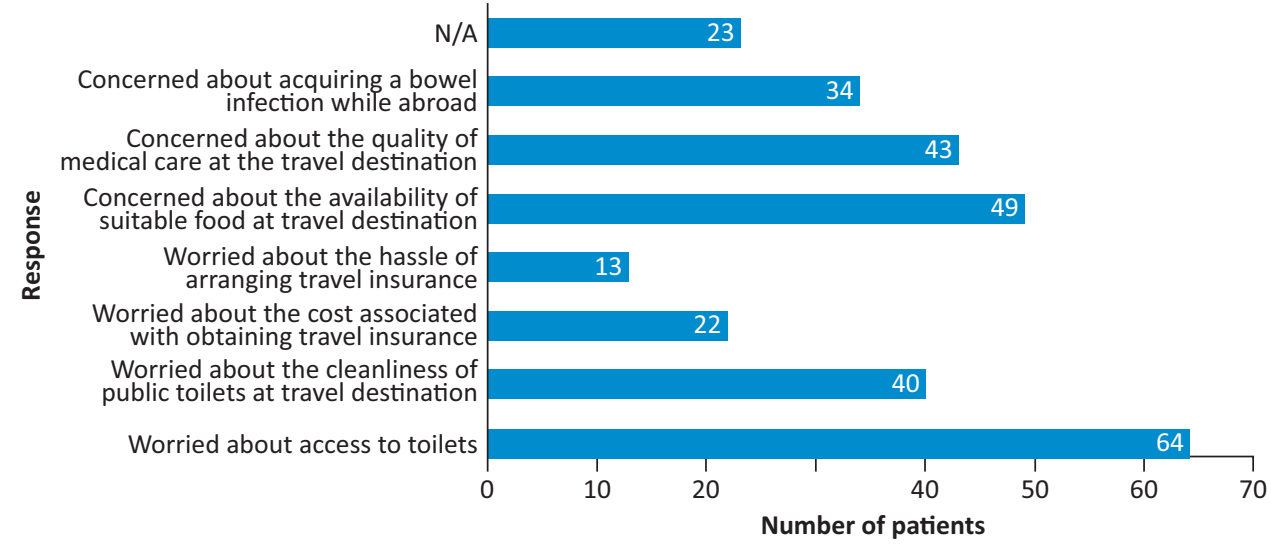

Fig 1. What is the main reason inflammatory bowel disease has affected your travel destination? recruited. Crohn's Disease was present in $54 \%$ of patients, $39 \%$ had ulcerative colitis, $5 \%$ were unsure of their type of IBD and $2 \%$ had indeterminate colitis. From the sample, 56.6\% were taking 5-aminosalicylates (5-ASAs), 52.9\% were on immunosuppressant medication and $20.6 \%$ were receiving monoclonal antibodies. Patients in this study were given a diagnosis of IBD from less than 1 to over 60 years ago and the mean time since diagnosis was 12.2 years. Over $89 \%$ had been abroad since being diagnosed. The main reasons for travel were leisure (77\%) and work (19.9\%). Thirty percent reported that having IBD limited travel overseas and $40 \%$ claimed it affected their choice of travel destination. Figure 1 shows how IBD affects patient's choice of travel destination.

Sixty-one percent of patients worried about facing problems pertaining to healthcare if they travelled abroad. Seven percent reported being refused health insurance outright and $47 \%$ travelled had abroad without health insurance since being diagnosed with IBD. Nine percent admitted that if they travelled abroad without health insurance, the reason for this was due to their IBD. Sixtyfour percent felt that they received adequate medical advice from their doctor prior to their departure from the UK, while $21 \%$ were dissatisfied with their advice; seventy-eight percent were interested in receiving advice from their doctor prior to their travel in the future. Sixty percent were unaware that taking immunosuppressant medication could affect the type of vaccinations they receive. Sixty-three percent claimed their doctor did not inform them that they should avoid live vaccines if they are on immunosuppressant medication; $12 \%$ actually received a live vaccine such as the yellow fever vaccine, prior to their travel abroad.

Thirty-eight percent of patients who travelled were either experiencing a flare-up at the time or recovering from one and $18 \%$ travelled despite being within one week of a flare-up. Ninety-two percent were unaware that travelling to high-altitude destinations could precipitate flare-ups and $27 \%$ had travelled to a high-altitude destination of which $46 \%$ experienced a flare-up while travelling or within 4 weeks of travel. In this study, $72 \%$ were unaware of the 'Can't wait' card and over $96 \%$ had never heard about the 'IBD passport' website. There were felt to be a number of simple measures by which the IBD clinic could assist in travel plans with the commonest suggestion being medical support to obtain insurance (Fig 2).

Despite the risks, travel abroad is frequent among patients with IBD. Over $89 \%$ of patients in this study had chosen to travel abroad regardless of any associated health risk. We identified five different ways in which doctors could help IBD patients prior to their travel. Many patients felt if their doctor provided them with more support before their travel, such as a written management plan, or a written/electronic prescription to take abroad, this would help them. These suggestions are shown in Fig 3.

The internet is the most widely used source of pre-travel information followed closely by IBD specialist doctors. The majority of patients $(68 \%)$ use the internet, IBD specialist doctor or IBD nurse for pre-travel information (Fig 4). GPs are the primary point of call for patients to obtain pre-travel advice due to the fact

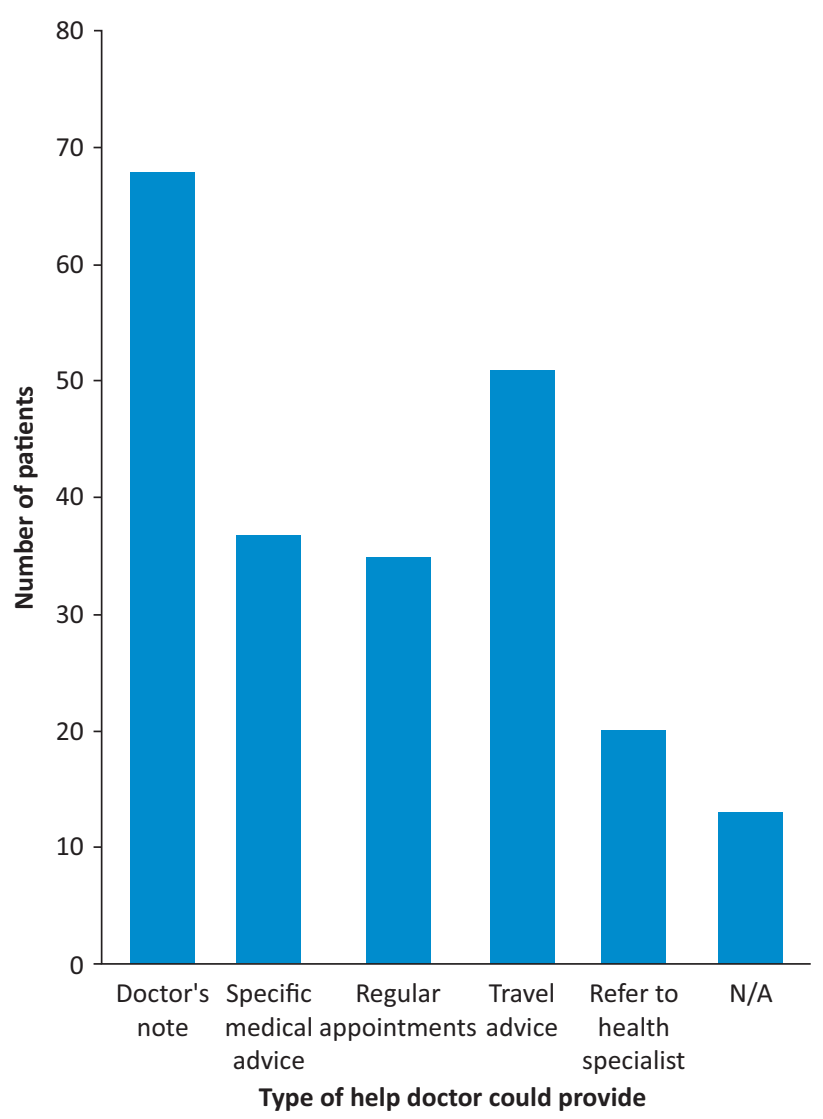

Fig 2. Graph to show patient responses to the question: how do you feel your doctor could help you secure health insurance? 


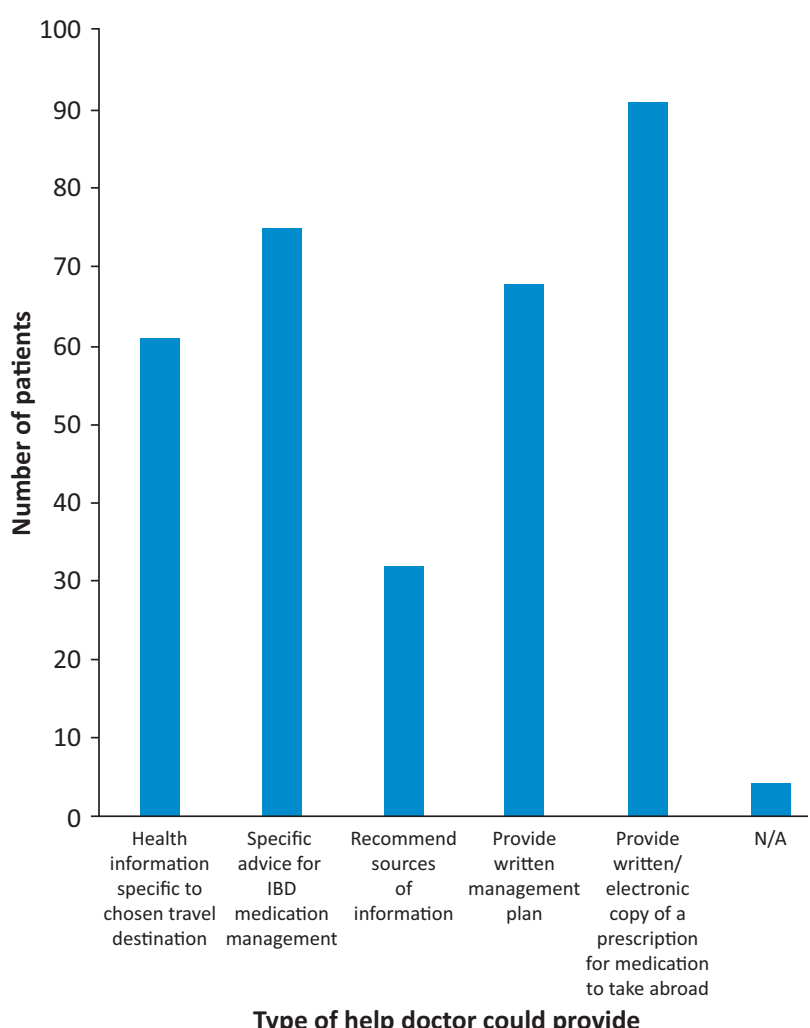

Fig 3. Graph to show patient responses to the question: how would you feel your doctor could help you the most before your travel abroad?

that IBD specialist doctor and nurse appointments are usually booked on 3-monthly or 6-monthly bases which rarely take into account patient's travel plans. Despite this, our research shows that GPs are poorly utilised for pre-travel information with only

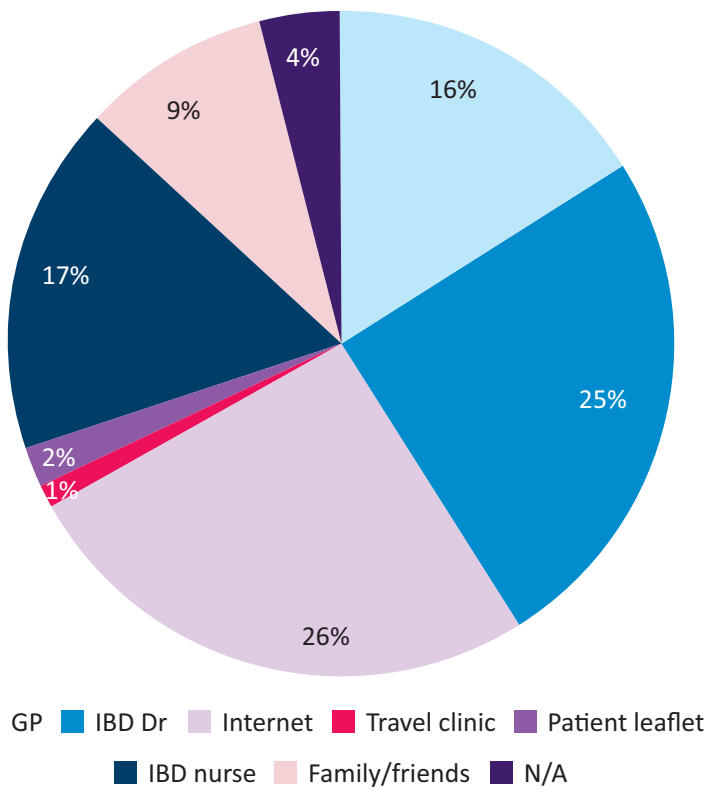

Fig 4. Most useful pre-travel information services. IBD = inflammatory bowel disease
$16 \%$ of patients using them. Travel clinics and patient leaflets are almost entirely unused, with only $1 \%$ and $2 \%$ of patients respectively reporting their usefulness.

In this survey over $55 \%$ of the patients who were uninformed about the need to avoid live vaccines were on immunosuppressants.

\section{Discussion}

This survey has identified that many IBD patients are being refused health insurance and most have to pay higher premiums which could explain why many chose to travel abroad with no health insurance. Health insurance companies require declarations of chronic conditions such as IBD which is associated with higher costs. ${ }^{5}$ Some IBD patients, albeit a minority, are still refused health insurance when they travel abroad. Encouragingly, only one patient in our study had ever been refused health insurance to the USA, whereas 10 had been refused health insurance when travelling to any foreign destination. These figures could suggest that although health insurance is still a problem for some patients with IBD, ${ }^{6}$ it is not as much of a problem as it once was.

Many IBD patients travelled abroad despite experiencing or recovering from recent flare-ups. This study confirms the idea that despite an increased health risk due to travelling, most patients tend to travel anyway which supports previous research also showing this trend. ${ }^{7}$ IBD continues to limit the travel of a considerable number of patients (30\%) and affected the choice of travel destination in $40 \%$ of patients. A multitude of reasons exist for how IBD could affect patients' choice of travel destination and we identified seven possible reasons for this.

The majority of IBD patients worry they will encounter problems related to their healthcare if they travel abroad. This shows travelrelated anxiety is a significant problem for IBD patients and more needs to be done to reduce their worries. In conditions such as IBD, where stress can exacerbate symptoms, precipitate flare-ups and cause relapses, it is especially important that these travel-related worries do not contribute to psychological stress. ${ }^{8}$

European guidelines from the European Crohn's and Colitis organisation suggest that travel advice should be provided jointly by a specialist gastroenterologist and an expert travel clinic. ${ }^{9}$ However, in the UK no such national guidelines exist and the burden of responsibility lies predominantly with GPs to provide appropriate travel advice to patients. Travel clinics do exist but travel medicine is a relatively new field which is yet to be established here in the UK. ${ }^{10}$ Our research has shown that a considerable number of patients felt that they did not receive adequate medical advice prior to their travel. Furthermore, the vast majority of patients were interested in receiving medical advice prior to their travel abroad which reiterates that the current level of travel advice is insufficient. Travel clinics are not used here in the UK, as suggested in previous literature. ${ }^{11}$

Despite national campaigns by the Irritable Bowel Syndrome (IBS) network such as the 'Can't wait' card, our study revealed that $72 \%$ of patients were unaware of its existence. The 'Can't wait' card is a discreet method of communicating the need for help to members of staff in public places and shops to enable quicker access to toilets. ${ }^{12}$ This service is useful for patients not just within the UK but travelling abroad as well, since it is available in various international formats in many different languages. A similar service is available via the IBD Passport website which provides IBD specific travel / vaccination advice, mobile phone apps, ways of communicating medical information while travelling 
and guided instructions for specific IBD travel related issues. ${ }^{13}$ This evidence-based service was created by an IBD specialist nurse and is verified by IBD patients to be an invaluable companion to their travel abroad. Unfortunately, almost all patients in this study were completely unaware of this service. In order to provide better travel advice to patients it is imperative that we fully utilise pre-existing services such as the 'Can't wait' card and the IBD Passport.

In 2013, the National Key Scheme (NKS) was launched which provides a guide that includes the location of over 9,000 NKS toilets, in partnership with RADAR key and Disability Rights UK. These toilets are privately available for patients who have a medical condition which requires the need for a toilet. They are found in shopping centres, pubs, cafes, department stores, bus and train stations and many other locations around the country and are accessed with the RADAR key. ${ }^{14}$ There is now also a smartphone application which locates these toilets on a map for patients to access. ${ }^{15}$ This study did not measure the awareness patients had of this service, but future projects should endeavour to continue raising awareness.

Previous research shows travel to high-altitude destinations may precipitate flare-ups in IBD patients due to a hypoxia-induced inflammatory mechanism of action. ${ }^{16}$ Most patients in this study, were unaware of this potential IBD specific health risk. Our survey showed a significant number of patients experienced a flareup following their travel abroad to a high-altitude destination, suggesting that more research is needed in the area of highaltitude IBD travel. Despite an unclear aetiology, if this trend becomes increasingly common perhaps it would be appropriate for doctors to advise higher-risk patients to avoid travelling to high-altitude destinations prophylactically.

There are several limitations to this study. Namely, not all patients in this study actually travelled abroad $(11 \%$ did not travel abroad). Many patients reported that IBD did not limit their travel abroad and for the majority of patients $(60 \%)$ their IBD did not affect their choice of travel destination. This could be because travel simply does not have as great of an effect on IBD morbidity as it was originally thought to. Another explanation could be that patients who feel relatively uninhibited by their IBD have a good control of their condition and this may not be the case for patients who have poor control of their condition.

Single-centre experience is a limitation as patient demographics change elsewhere in London, or even anywhere in the UK. Other limitations include the method of convenience sampling which is vulnerable to selection bias and sampling errors. The questionnaire survey in general has several limitations. For instance, questions can be interpreted differently according to different patients, emotions and behaviour contribute to answers, and there is no way of telling how truthful patients are in their responses. There is also a level of researcher imposition in developing the questionnaire which inevitably means that some key areas could be missed out altogether.

Although some patients report IBD as the reason for their inability to secure health insurance, this may not be the case for everyone. Some people just do not worry about their health and would not purchase health insurance regardless of their IBD. There is another subset of the population who might always travel abroad uninsured because the quantity of travel done is limited. For these patients the risk of falling sick abroad and incurring further medical costs is a transient risk that may not appear worth the cost or might even be unaffordable. It would be useful to screen patients according to socioeconomic group as these behaviours might be more common in less affluent patients. The reasons behind the feeling of being inadequately informed about travel and vaccinations are likely to be complex and not assessed by the questionnaire.

It is recommended that IBD patients on immunosuppressant medication should avoid live vaccines altogether. ${ }^{11}$ Previous studies have shown that most patients are unaware of this vital information. ${ }^{17}$ Our survey population mirrored this. While this study explores the medication that patients are currently on, which allows us to determine if they are immunosuppressed or not, it does not give us the whole picture. For instance, a caveat in this study is there could be patients in this group who are not on immunosuppressant medication but have only recently been taken off them. It is recommended that patients remain off immunosuppressant medication for at least 3 months before taking any live vaccines. ${ }^{18}$ Future questionnaires should consider this requirement and screen if patients have been given this adequate buffer period to regain an efficient immune system.

\section{Conclusion}

Overall, our survey shows several ways in which IBD patients are adversely affected by travel abroad. Acquiring health insurance in this subgroup of patients is an ongoing problem with over a quarter of patients travelling abroad without adequate health insurance. Many admit the reason for travelling uninsured is due to their IBD; therefore, more needs to be done in supporting these patients to secure health insurance prior to their travel. The survey has identified some simple steps that IBD physicians can take to help.

Pre-travel health advice needs to be improved. One big area of concern is that an alarmingly high number of patients are uninformed of their need to avoid live vaccines while being on immunosuppressants and subsequently wrongly receive these vaccinations. This poses a major health risk in IBD patients and causes avoidable flare-ups.

Although the internet remains the most popular source of pre-travel information, already established online sources such as the IBD Passport and 'Can't wait' card remain unknown to most patients. Future endeavours should focus on increasing awareness of these services as well as providing more information regarding new services such as the RADAR key. ${ }^{19}$

Finally, a large number of patients suffered a flare-up following travel to a high-altitude destination which supports previous literature on the effects of hypoxia-induced inflammation in patients with IBD. ${ }^{13}$ This warrants further research that could potentially be used for public health recommendations/policy. It might be useful for patients to have access to an information leaflet providing specific IBD travel advice which could be the focus of future patient service improvement projects.

\section{References}

1 Ben-Horin S, Bujanover Y, Goldstein S et al. Travel associated health risks for patients with inflammatory bowel disease. Clin Gastroenterol Hepatol 2012;10:160-5.

2 Rahier JF, Magro F, Abreu C et al. Second European evidence based consensus on the prevention, diagnosis and management of opportunistic infections in inflammatory bowel disease. ] Crohns Colitis 2014;8:443-68.

3 Rahier JF, Moutschen M, Van Gompel A et al. Vaccinations in patients with immune-mediated inflammatory diseases. Rheumatology 2010;49:1815-27. 
4 Health Research Authority. National Research Ethics Service: www.hra.nhs.uk/research-community/before-you-apply/determinewhether-your-study-is-research/ [Accessed 4 June 2017].

5 Russel MG, Ryan BM, Dagnelie PC et al. Insurance problems among inflammatory bowel disease patients: results of a Dutch population based study. Gut 2003;52:358-62.

6 IBD patients face insurance and financial burdens. Crohn's \& Colitis Foundation, 2017 www.crohnscolitisfoundation.org/news/ ibd-patients-face-insurance.html [Accessed 4 June 2017].

7 Greveson K, Shepherd T, Hamilton M, Murray C. P574. A recent flare of disease does not prohibit travel: early results of a single centre study in inflammatory bowel disease and travel. J Crohns Colitis 2014;8:S307-8.

8 Mawdsley JE, Rampton DS. Psychological stress in IBD: new insights into pathogenic and therapeutic implications. Gut 2005;54:1481-91.

9 Rahier J, Magro F, Abreu C et al. Second European evidence-based consensus on the prevention, diagnosis and management of opportunistic infections in inflammatory bowel disease. J Crohns Colitis 2014;8:443-68.

10 Greveson K, Shepherd T, Mulligan ] et al. Travel health and pretravel preparation in the patient with inflammatory bowel disease. Frontline Gastroenterology 2015;7:60-5.

11 Dezfoli S, Melmed GY. Vaccination issues in patients with inflammatory bowel disease receiving immunosuppression. Gastroenterol Hepatol 2012:504-12.
12 Can't Wait Card, The IBS Network. www.theibsnetwork.org/cant-waitcard/ [Accessed 2 June 2017].

13 IBD Passport. http://ibdpassport.com [Accessed 2 June 2017].

14 Disability Rights UK. Genuine Radar Key. www.disabilityrightsuk. org/shop/genuine-radar-key [Accessed 28 February 2018].

15 Colitis UK. Public Toilets. www.ulcerativecolitis.org.uk/Public_Toilets.htm [Accessed 28 February 2018].

16 Vavricka S, Rogler G, Maetzler S et al. High altitude journeys and flights are associated with an increased risk of flares in inflammatory bowel disease patients. J Crohns Colitis 2014;8:191-9.

17 Rahier J, Moutschen M, Van Gompel A et al. Vaccinations in patients with immune-mediated inflammatory diseases. Rheumatology 2010;49:1815-27.

18 Reich J, Wasan S, Farraye FA. Vaccinating patients with inflammatory bowel disease. Gastroenterol Hepatol 2016;12:540-6.

19 Colostomy Association. RADAR - National Key Scheme. www.colostomyuk.org/information/using-accessible-toilets/ [Accessed 4 June 2017].

Address for correspondence: Dr Varun Philip, St George's Hospital, Gastroenterology, Blackshaw Road, London SW17 OQT, UK.

Email: m0901253@sgul.ac.uk 